\title{
Отжиг высоковольтных 4H-SiC диодов Шоттки, облученных электронами при высокой температуре
}

\author{
(C) А.А. Лебедев ${ }^{1}$, В.В. Козловскийㅁ, М.Е. Левинштейн ${ }^{1}$, Д.А. Малевский ${ }^{1}$, \\ Г.А. Оганесян ${ }^{1}$, А.М. Стрельчук ${ }^{1}$, К.С. Давыдовская ${ }^{1}$ \\ ${ }^{1}$ Физико-технический институт им. А.Ф. Иоффре Российской академии наук, \\ 194021 Санкт-Петербург, Россия \\ ${ }^{2}$ Санкт-Петербургский политехнический университет Петра Великого, \\ 195251 Санкт-Петербург, Россия \\ IE-mail: melev@nimis.ioffe.ru
}

Поступила в Редакцию 30 ноября 2021 г.

В окончательной редакции 19 декабря 2021 г.

Принята к публикации 19 декабря 2021 г.

\begin{abstract}
Впервые исследовано влияние отжига на параметры $4 H$-SiC диодов Шоттки, облученных электронами при высокой температуре. Энергия электронов составляла $0.9 \mathrm{MэB}$, облучение проводилось при температурах 23 , 300 и $500^{\circ} \mathrm{C}$ в диапазоне флюенсов $1 \cdot 10^{16}-1.3 \cdot 10^{17} \mathrm{~cm}^{-2}$. Результаты отжига образцов, облученных при высоких температурах, качественно отличаются от результатов отжига образцов, облученных тем же флюенсом при комнатной температуре. Полученные результаты свидетельствуют о том, что при высокотемпературном („горячем“) облучении спектр вводимых в $\mathrm{SiC}$ радиационных дефектов существенно отличается от спектра дефектов, вводимых при комнатной температуре. При больших значениях флюенса при температурах облучения 300 и $500^{\circ} \mathrm{C}$ обнаружен эффект „обратного отжига“, когда сопротивление базы диода не падает, а возрастает в результате отжига.
\end{abstract}

Ключевые слова: карбид кремния, диоды Шоттки, электронное облучение, отжиг, электрические свойства.

DOI: 10.21883/FTP.2022.04.52201.9777

\section{1. Введение}

Высоковольтные 4H-SiC диоды Шоттки с блокирующим напряжением 600-1700 В с каждым годом все шире используются в автомобильной и космической электронике, оборудовании ядерных электростанций, коммутаторах солнечных преобразователей большой площади, системах коррекции коэффициентов мощности, в устройствах питания компьютеров и т.д. [1-4]. Устойчивость приборов на основе любого материала по отношению к различным типам излучения часто служит одним из основных критериев, определяющих возможность их использования, особенно в таких применениях, как космическая и авиационная электроника, ускорители заряженных частиц и электронное оборудование ядерных реакторов.

Влияние на свойства высоковольтных $4 H-\mathrm{SiC}$ диодов Шоттки облучения электронами высокой энергии и последующего отжига исследовалось в целом ряде работ (см., например, [5-12] и ссылки в этих работах). Все эти исследования проводились в условиях, когда диоды Шоттки облучались при комнатной температуре. Недавно в работе [13] было впервые исследовано влияние электронного облучения, проделанного при повышен-

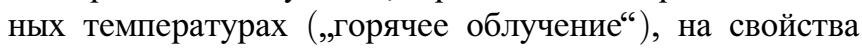
высоковольтных $4 H$-SiC диодов Шоттки. Было продемонстрировано, что радиационная стойкость приборов монотонно возрастает с возрастанием температуры облучения $T_{i}$. При этом, чем выше доза облучения, тем сильнее влияет температура при облучении на уровень радиационного повреждения диодов. В работе [13] было высказано предположение, что полученные при высокотемпературном облучении результаты, возможно, требуют некоторого пересмотра существующих концепций формирования радиационных дефектов в $4 H$-SiC $n$-типа.

В настоящей работе впервые исследованы особенности отжига $4 H$-SiC диодов Шоттки, облученных электронами при температурах 300 и $500^{\circ} \mathrm{C}$. Результаты сопоставлены с соответствующими данными, установленными при отжиге диодов Шоттки, облученных электронами при комнатной температуре.

\section{2. Условия эксперимента}

Исследовались диоды Шоттки CPW3-1700-S010BWP [14] с блокирующим напряжением 1700 В и средним значением выпрямленного тока 10 А. Диоды облучались электронами с энергией 0.9 МэВ в импульсном режиме. Длительность импульсов составляла 330 мкс, частота следования импульсов равнялась 490 Гц. Плотность тока электронного пучка была равной 12.5 мкА/см ${ }^{-2}$. Так как длина пробега электронов с энергией $0.9 \mathrm{MэB} \mathrm{в} \mathrm{SiC}$ coставляет $\sim 1.0$ мм при толщине базы диодов $L \approx 20$ мкм, дефекты вводились равномерно по объему образцов.

Высокотемпературное облучение диодов проводилось в специальной мишенной камере на воздухе. Для этого ускоренные электроны выводились через тонкую ( 50 мкм) титановую фольгу из вакуумного объема 
ускорителя. Облучение могло производиться при температуре мишени в диапазоне от 23 до $600^{\circ} \mathrm{C}$. Точность поддержания температуры равнялась $\pm 5^{\circ} \mathrm{C}$.

Параметры отжига были экспериментально выбраны так, чтобы после облучения, приводящего к увеличению сопротивления базы на порядок, исходное значение сопротивления восстанавливалось одиночным отжигом. Такому условию отвечал отжиг в атмосфере сухого азота при температуре $250^{\circ} \mathrm{C}$ в течение 60 мин.

Изотермические вольт-амперные характеристики (BAX) диодов измерялись при комнатной температуре. При достаточно высоком сопротивлении базы $\left(R_{b} \geq 10^{3} \mathrm{OM}\right)$ измерения производились на постоянном токе. При $R_{b} \leq 10^{3}$ Ом измерения проводились в режиме одиночных импульсов.

\section{3. Результаты и обсуждение}

При всех температурах облучения $T_{i}$, во всем диапазоне флюенсов $\Phi$ и при всех режимах отжига зависимость прямого тока от напряжения на экспоненциальном участке зависимости BAX практически не изменяются $[9,12,13]$. Параметром, наиболее чувствительным к температуре и дозе облучения, является сопротивление базы диода $R_{b}$.

На рис. 1 показаны прямые ВАХ необлученного диода, диода после облучения электронами с энергией $0.9 \mathrm{MэВ} \mathrm{при} \mathrm{температуре} T_{i}=300^{\circ} \mathrm{C}$ флюенсом $\Phi=1 \cdot 10^{16} \mathrm{~cm}^{-2}$ и после оптимизированного последующего отжига.

Измеренные величины дифференциального сопротивления (сопротивления базы $R_{b}$ ) составляют $R_{b}=0.1,1.22$ и 0.14 Ом для кривых 1,2 и 3 соответственно. Таким образом, облучение диодов приводит к росту $R_{b}$ в $\sim 12$ раз. Полагая подвижность электронов постоянной [15], следует сделать вывод, что в результате облучения в базе диода возник акцепторный уровень (уровни) с суммарной концентрацией, сопоставимой с исходной концентрацией электронов в базе необлученных диодов $n_{0} \approx 3.4 \cdot 10^{15} \mathrm{~cm}^{-3}$.

В работе [12] 1700 В диоды C3D10170H (Cree) облучались электронами с энергией $4.5 \mathrm{MэB} \mathrm{при} \mathrm{комнатной}$ температуре. При дозе 700 кГр, что при данной энергии электронов соответствует флюенсу $\Phi \approx 2.5 \cdot 10^{15} \mathrm{~cm}^{-2}$, концентрация акцепторного уровня $E 0 \quad\left(E_{c}-0.22\right.$ эВ) составила $4 \cdot 10^{15} \mathrm{~cm}^{-2}$.

В работе [6] образцы $4 H$-SiC $n$-типа с исходной концентрацией электронов $2 \cdot 10^{15} \mathrm{~cm}^{-3}$ облучались при комнатной температуре электронами с энергией 8.6 МэВ. Максимальный флюенс $\Phi$ равнялся $\approx 9.5 \cdot 10^{14} \mathrm{~cm}^{-2}$. При этом концентрация акцепторного уровня $S 2\left(E_{c}-0.39 \mathrm{\jmath} \mathrm{B}\right)$ составляла $4 \cdot 10^{14} \mathrm{~cm}^{-3}$. По мере отжига при температурах $\sim 360-400 \mathrm{~K}$ амплитуда пика $S 2$ снижалась, и параллельно росла концентрация электронов в образце.

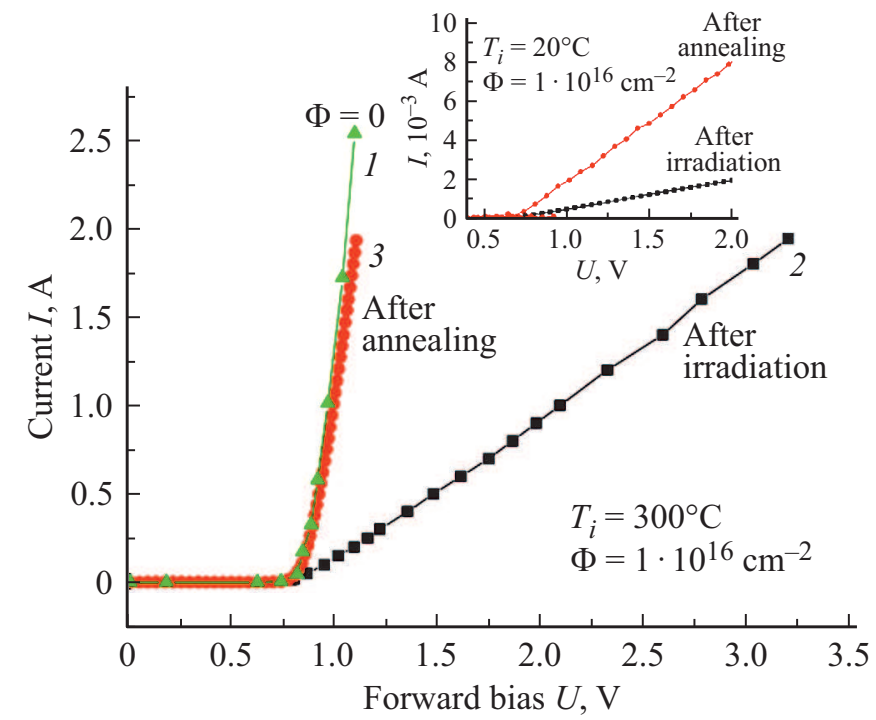

Рис. 1. Прямые BAX необлученного диода (кривая 1), после облучения электронами флюенсом $\Phi=1 \cdot 10^{16} \mathrm{~cm}^{-2}$ при температуре $T_{i}=300^{\circ} \mathrm{C}$ (кривая 2) и после последующего однократного отжига в атмосфере сухого азота в течение 60 мин при $250^{\circ} \mathrm{C}$ (кривая 3). На вставке показаны ВАХ диода после облучения при комнатной температуре тем же флюенсом $\Phi$ и последующего отжига в том же режиме.

Как видно из рис. 1, однократный отжиг в описанном выше режиме приводит к восстановлению концентрации электронов в базе до значения, в 1.4 раза меньшего, чем исходная концентрация электронов в базе необлученного диода.

Облучение диодов флюенсом $\Phi=1 \cdot 10^{16} \mathrm{~cm}^{-2}$ при комнатной температуре (см. вставку к рис. 1) приводит к значительно более сильному возрастанию сопротивления базы [13]. Величина $R_{b}$ после облучения составляет 640 Ом. Последующий отжиг в режиме, приводившем к практически полному восстановлению концентрации электронов в базе при облучении при $300^{\circ} \mathrm{C}$, несколько увеличивает концентрацию электронов в базе. Однако даже после отжига величина $R_{b}$ составляет $\sim 160$ Ом.

Такой результат, по-видимому, служит дополнительным аргументом в пользу необходимости некоторого пересмотра существующих концепций формирования радиационных дефектов в $4 H$-SiC $n$-типа при высокотемпературном облучении.

Увеличение флюенса до $\Phi=4.5 \cdot 10^{16} \mathrm{~cm}^{-2}$ (рис. 2) приводит к заметному росту сопротивления базы и к существенному изменению результатов отжига.

После облучения (кривая 2) дифференциальное сопротивление $R_{b}$, измеренное в интервале прямых напряжений $1 \leq U \leq 2 \mathrm{~B}$, составляет 48 Ом. Первый отжиг уменьшает сопротивление базы до значения $R_{b} \approx 3.8$ Ом. Таким образом, после первого отжига сопротивление остается в $\sim 40$ раз больше, чем сопротивление необлученного диода. Однако повторный отжиг в 


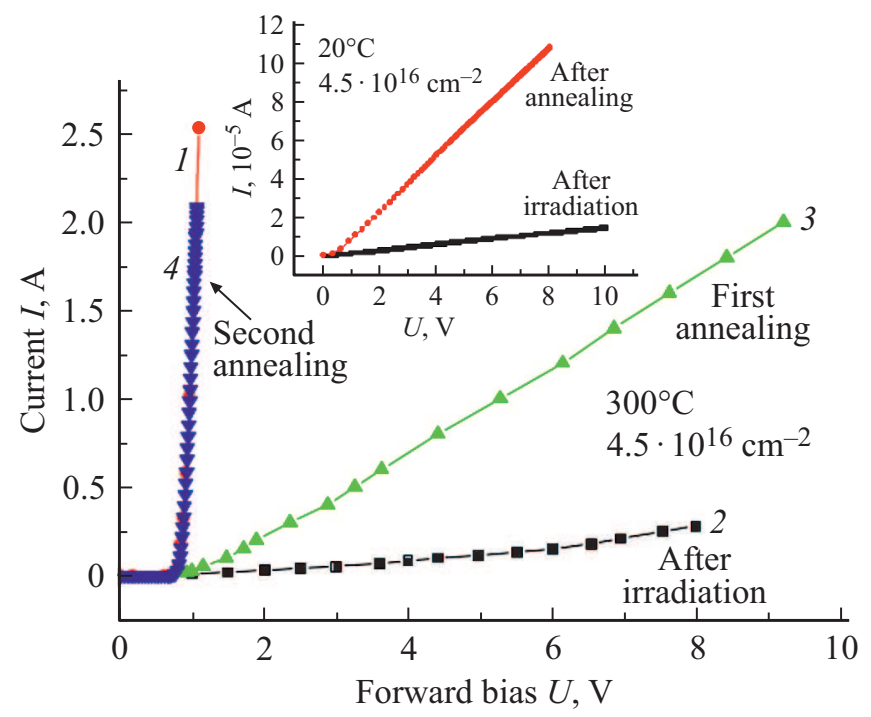

Рис. 2. Прямые ВАХ необлученного диода (кривая 1), после облучения электронами флюенсом $\Phi=4.5 \cdot 10^{16} \mathrm{~cm}^{-2}$ при температуре $T_{i}=300^{\circ} \mathrm{C}$ (кривая 2), после последующего однократного отжига в атмосфере сухого азота в течение 60 мин при $250^{\circ} \mathrm{C}$ (кривая 3) и после повторного отжига в том же режиме (кривая 4). На вставке показаны ВАХ диода после облучения при комнатной температуре тем же флюенсом $\Phi$ и однократного последующего отжига в том же режиме.

том же режиме приводит к практически полному восстановлению исходной концентрации электронов в базе диода. Сопротивление базы после повторного отжига $R_{b} \approx 0.11 \mathrm{Oм}$.

После облучения флюенсом $\Phi=4.5 \cdot 10^{16} \mathrm{~cm}^{-2}$ при комнатной температуре (см. вставку к рис. 2) дифференциальное сопротивление $R_{b}$, измеренное в интервале прямых напряжений $1 \leq U \leq 2 \mathrm{~B}$, равняется $\approx 7.4 \cdot 10^{5}$ Ом. После отжига сопротивление уменьшается до значения $R_{b} \approx 6.7 \cdot 10^{4}$ Ом.

При дальнейшем увеличении флюенса полное восстановление исходной концентрации электронов, повидимому, становится невозможным (рис. 3 ).

После облучения дифференциальное сопротивление $R_{b}$, измеренное в интервале прямых напряжений $1 \leq U \leq 2 \mathrm{~B}$, составляет 270 Ом (см. вставку к рис. 3). Первый отжиг уменьшает величину $R_{b}$ до значения $R_{b} \approx 3.6$ ОМ, практически равного значению $R_{b}$ после первого отжига после облучения флюенсом $\Phi=4.5 \cdot 10^{16} \mathrm{~cm}^{-2}$ (рис 2, кривая 3). Однако повторный отжиг приводит не к уменьшению, а к некоторому росту $R_{b}$ до значения $R_{b} \approx 4.3$ Ом. Из этого факта следует очевидный вывод: эффект увеличения флюенса не сводится просто к росту концентрации вводимых дефектов.

На рис. 4 представлены результаты, полученные при облучении диода тем же флюенсом, что и на рис. 3 , но при температуре облучения $T_{i}=500^{\circ} \mathrm{C}$.
Дифференциальное сопротивление $R_{b}$, измеренное в интервале прямых напряжений $1 \leq U \leq 2 \mathrm{~B}$, составляет в этом случае 1.54 Ом. Первый отжиг несколько уменьшает величину $R_{b}$ до значения $R_{b}=1.43$ Ом. После второго и третьего отжига значения $R_{b}$ составили 3.4 и 4.5 Ом соответственно.

Как отмечалось в работе [16], нагрев $4 H-\mathrm{SiC}$ диодов Шоттки до температур, превышающих $370^{\circ} \mathrm{C}$, приводит к диффузии и частичному вплавлению металла контактов (никель) в поверхность $\mathrm{SiC}$. Следует, однако, заме-

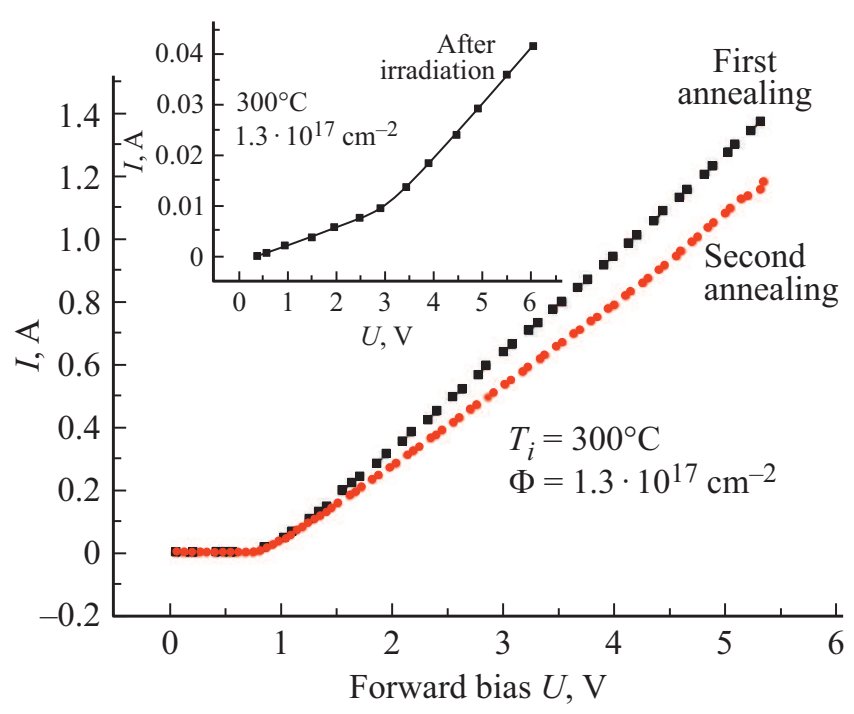

Рис. 3. Прямые ВАХ после облучения электронами флюенсом $\Phi=1.3 \cdot 10^{17} \mathrm{~cm}^{-2}$ при температуре $T_{i}=300^{\circ} \mathrm{C}$ (см. вставку), после последующего отжига в атмосфере сухого азота в течение 60 мин при $250^{\circ} \mathrm{C}$ и после повторного отжига в том же режиме.

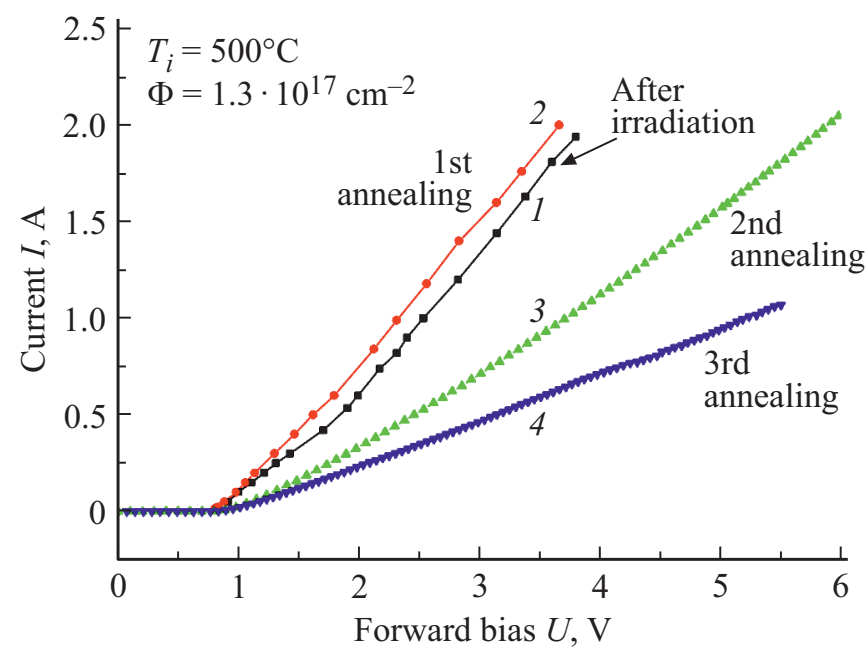

Рис. 4. Прямые BAX после облучения электронами флюенсом $\Phi=1.3 \cdot 10^{17} \mathrm{~cm}^{-2}$ при температуре $T_{i}=500^{\circ} \mathrm{C}$ (кривая 1), после последующего отжига в атмосфере сухого азота в течение 60 мин при $250^{\circ} \mathrm{C}$ (кривая 2), после повторного отжига в том же режиме (кривая 3) и после третьего отжига (кривая 4). 
тить, что зависимость тока от напряжения при малых смещениях (экспоненциальный участок) не зависела от $T_{i}$ и после облучения при температуре $500^{\circ} \mathrm{C}$ следовала обычной зависимости $I=I_{s}[\exp (q U / n k T)-1]$. При этом коэффициент идеальности $n$ оставался таким же, как и в необлученных диодах [13].

Далее, чтобы убедиться, что в процессе, включающем нагрев образцов до температуры $500^{\circ} \mathrm{C}$, стабилизация температурного режима в камере и охлаждение не влияют на BAX, образцы нагревались (без облучения) в том же режиме нагрева и охлаждения, что и в экспериментах по облучению. Во всем диапазоне смещений ВАХ после такого температурного стресса совпадали с исходными $I-V$-характеристиками с точностью до толщины линии.

Полученные результаты подтверждают высказанное ранее предположение о том, что при высокотемпературном („горячем“) облучении возникают дефекты, отсутствующие при облучении при комнатной температуре. При относительно небольших значениях флюенсов дефекты, введенные при $300^{\circ} \mathrm{C}$, отжигаются практически полностью после отжига при температуре $250^{\circ} \mathrm{C}$, в то время как при таком же режиме отжига введенные облучением при комнатной температуре дефекты отжигаются лишь в очень незначительной степени (рис. 1 и 2). С ростом дозы горячего облучения появляется, повидимому, вторая группа более сложных радиационных комплексов. Результатом распада таких комплексов при отжиге является образование точечных дефектов, обладающих свойствами глубоких акцепторных ловушек. Этим может объясняться так называемый „обратный отжиг“ $[17,18]$, при котором сопротивление базы возрастает, а не убывает (рис. 3). Повышение температуры облучения до $500^{\circ} \mathrm{C}$ (рис. 4) приводит, по-видимому, к еще более эффективному смещению радиационного дефектообразования в сторону комплексообразования. При этом „обратный отжиг“ оказывается значительно более выраженным (рис. 4).

\section{4. Заключение}

Впервые исследовано влияние отжига на параметры $4 H$-SiC диодов Шоттки, облученных электронами при высокой температуре. Энергия электронов составляла $0.9 \mathrm{MэВ,} \mathrm{облучение} \mathrm{проводилось} \mathrm{при} \mathrm{тем-}$ пературах 23, 300 и $500^{\circ} \mathrm{C}$ в диапазоне флюенсов $1 \cdot 10^{16}-1.3 \cdot 10^{17} \mathrm{~cm}^{-2}$. Результаты отжига образцов, облученных при высоких температурах, качественно отличаются от результатов отжига образцов, облученных тем же флюенсом при комнатной температуре. Полученные результаты свидетельствуют о том, что при высокотемпературном (,горячем“6) облучении спектр вводимых в $\mathrm{SiC}$ радиационных дефектов существенно отличается от спектра дефектов, вводимых при комнатной температуре. При больших значениях $\Phi$ при температурах облучения 300 и $500^{\circ} \mathrm{C}$ обнаружен эффект „обратного отжига“, когда сопротивление базы диода не падает, а возрастает в результате отжига.

\section{Конфликт интересов}

Авторы заявляют, что у них нет конфликта интересов.

\section{Список литературы}

[1] T. Nakamura, M. Sasagawa, Y. Nakano, T. Otsuka, M. Miura. Int. Power Electron. Conf. (Sapporo, 2010).

[2] Q. Xun, B. Xun, Z. Li, P. Wang, Z. Cai. Renew. Sust. Energ. Rev., 70, 1336 (2017).

[3] B.J. Baliga. 76th Device Research Conf. (DRC) (Santa Barbara, 2018). doi: 10.1109/drc.2018.8442172

[4] А.А. Лебедев, П.А. Иванов, М.Е. Левинштейн, Е.Н. Мохов, С.С. Нагалюк, А.Н. Анисимов, П.Г. Баранов. УФН, 189 (8), 803 (2019)

[5] G. Alfieri, E.V. Monakhov, B.G. Svensson, A. Hallén. J. Appl. Phys., 98, 113524 (2005).

[6] A. Castaldini, A. Cavallini, L. Rigutti, F. Nava. Appl. Phys. Lett., 85, 3780 (2004).

[7] P. Hazdra, Jan Vobecký. Phys. Status Solidi A, 216, 1900312 (2019).

[8] H. Kaneko, T. Kimoto. Appl. Phys. Lett., 98, 262106 (2011).

[9] V.V. Kozlovski, A.A. Lebedev, M.E. Levinshtein, S.L. Rumyantsev, J.W. Palmour. Appl. Phys. Lett., 110, 083503 (2017).

[10] О.М. Корольков, В.В. Козловский, А.А. Лебедев, Н. Слепчук, J. Toompuu, Т. Rang. ФТП, 53 (7), 991 (2019).

[11] E. Omotoso, W.E. Meyer, F.D. Auret, A.T. Paradzah, M. Diale, S.M.M. Coelho, P.J. Janse van Rensburg. Mater. Sci. Semicond. Process., 39, 112 (2015).

[12] J. Vobecký, P. Hazdra, S. Popelka, R.K. Sharma. IEEE Trans. Electron Dev., 62 (6), 1964 (2015).

[13] A.A. Lebedev, V.V. Kozlovski, M.E. Levinshtein, A.E. Ivanov, K.S. Davydovskaya, V.S. Yuferev, A.V. Zubov. Radiat. Phys. Chem., 185, 109514 (2021).

[14] https://www.digchip.com/datasheets/parts/datasheet/2101/ CPW3-1700-S010B-WP.php

[15] M.E. Levinshtein, S.L. Rumyantsev, M.S. Shur (eds). Properties of Advanced Semiconductor Materials: GaN, AIN, InN, BN, SiC, SiGe (John Wiley \& Sons Inc., N. Y., 2001).

[16] R. Karsthof, M.E. Bathen, A. Galeckas, L. Vines. Phys. Rev. B, 102, 18411 (2020). https://doi.org/10.1103/PhysRevB.102.184111

[17] T. Schulz, H. Feick, E. Fretwurst, G. Lindstrom, M. Moll, K.H. Mahlmann. IEEE Trans. Nucl. Sci., 41 (4), 791 (1994)

[18] S.J. Moloi, M. McPherson. Nucl. Instr. Meth. Phys. Res. Section B: Beam Interact. Mater. At., 440, 64 (2019).

Редактор А.Н. Смирнов 


\title{
Annealing of high voltage $4 H$-SiC \\ Schottky diodes irradiated with electrons at high temperatures
}

\author{
A.A. Lebedev ${ }^{1}$, V.V. Kozlovski ${ }^{2}$, M.E. Levinshtein ${ }^{1}$, \\ D.A. Malevsky ${ }^{1}$, G.A. Oganesyan ${ }^{1}$, \\ A.M. Strel'chuk ${ }^{1}$, K.S. Davydovskaya ${ }^{1}$ \\ ${ }^{1}$ loffe Institute, \\ 194021 St. Petersburg, Russia \\ ${ }^{2}$ Department of Experimental Physics, \\ Peter the Great St. Petersburg Polytechnic University, \\ 195251 St. Petersburg, Russia
}

Abstract The effect of annealing on the parameters of $4 H$-SiC Schottky diodes irradiated with electrons at high temperatures has been studied for the first time. The electron energy was $0.9 \mathrm{MeV}$. The irradiation was carried out at temperatures of 23,300 , and $500^{\circ} \mathrm{C}$ at fluences $\Phi$ in the range $1 \cdot 10^{16}-1.3 \cdot 10^{17} \mathrm{~cm}^{-2}$. The results of annealing after the irradiation at high temperatures are qualitatively different from the results of annealing after conventional irradiation at room temperature with the same fluencies. The results obtained indicate that the spectrum of radiation defects introduced into $\mathrm{SiC}$ under a high-temperature („hot“) irradiation differs significantly from that of defects introduced by irradiation at room temperature. At irradiation temperatures of 300 and $500^{\circ} \mathrm{C}$ and large $\Phi$, the effect of „reverse annealing“ was revealed, when the base resistance grows rather than falling as a result of annealing. 